\title{
A Generalisation of Dyson's Integration Theorem for Determinants
}

\author{
G. Akemann and L. Shifrin \\ Department of Mathematical Sciences \& BURSt Research Centre \\ School of Information Systems, Computing and Mathematics \\ Brunel University West London \\ Uxbridge UB8 3PH, United Kingdom
}

\begin{abstract}
Dyson's integration theorem is widely used in the computation of eigenvalue correlation functions in Random Matrix Theory. Here we focus on the variant of the theorem for determinants, relevant for the unitary ensembles with Dyson index $\beta=2$. We derive a formula reducing the $(n-k)$-fold integral of an $n \times n$ determinant of a kernel of two sets of arbitrary functions to a determinant of size $k \times k$. Our generalisation allows for sets of functions that are not orthogonal or bi-orthogonal with respect to the integration measure. In the special case of orthogonal functions Dyson's theorem is recovered.
\end{abstract}




\section{Motivation}

Random Matrix Theory (RMT) has many applications in all areas of Physics and beyond (see e.g. the introduction of [1]). For the class of invariant RMT Dyson's integration theorem is at the heart of the method of orthogonal polynomials when computing all eigenvalue correlation functions exactly, for finite $n \times n$ matrices. The resulting expressions are then amenable to the large- $n$ limit, in which universal RMT predictions follow. In the following we restrict ourselves to the integration theorem for determinants. Before presenting our generalisation thereof we briefly recall how it reveals all eigenvalue correlations in the unitary ensembles.

We start by stating Dyson's integration theorem, as cited in [1] (Theorem 5.1.4). Given $K(x, y)$ is a real valued function satisfying the following self-contraction property:

$$
\begin{aligned}
& \int d y K(x, y) K(y, z)=K(x, z), \\
& \int d y K(y, y)=c .
\end{aligned}
$$

Then it hold: 1

$$
\int d x_{1} \operatorname{det}_{1 \leq i, j \leq n}\left[K\left(x_{i}, x_{j}\right)\right]=(c-n+1) \operatorname{det}_{2 \leq i, j \leq n}\left[K\left(x_{i}, x_{j}\right)\right],
$$

thus reducing the size of the determinant by one through the integration. The theorem also holds for kernels of orthogonal polynomials in the complex plane or for bi-orthogonal polynomials. A similar statement is true for quaternion valued kernels with the determinant replaced by a quaternion determinant (or Pfaffian). We refer to [1] for details as we will only consider the ordinary determinant case here. Iterating the integration theorem the following holds for an $(n-k)$-fold integral:

$$
\int \prod_{l=1}^{n-k} d x_{l} \operatorname{det}_{1 \leq i, j \leq n}\left[K\left(x_{i}, x_{j}\right)\right]=(c-n+1) \ldots(c-k) \operatorname{det}_{n-k+1 \leq i, j \leq n}\left[K\left(x_{i}, x_{j}\right)\right] .
$$

It is this form that we will generalise as it is most useful when computing correlation functions in RMT. We emphasise that on the right hand side (rhs) the determinant has reduced to size $k \times k$ over the same kernel.

The application of eq. (1.3) to the unitary ensembles goes as follows. Suppose we have a set of orthogonal polynomials $p_{k}(x)=x^{k}+O\left(x^{k-1}\right)$ of order $k$ in monic normalisation satisfying

$$
\int d x w(x) p_{k}(x) p_{j}(x)=h_{k} \delta_{k j}
$$

Here $w(x)$ is a positive weight function such that all moments exist. From the polynomials we can construct orthonormal wave functions

$$
\varphi_{k}(x) \equiv h_{k}^{-1 / 2} w(x)^{1 / 2} p_{k}(x)
$$

and the following kernel

$$
K_{n}(x, y) \equiv \sum_{j=0}^{n-1} \varphi_{j}(x) \varphi_{j}(y)
$$

\footnotetext{
${ }^{1}$ The symmetry property $K(x, y)=K(y, x)$ stated in [1] is not necessary, as can be seen from the proof there.
} 
It satisfies Dyson's theorem above with $c=n$. In the symmetry class of unitary invariant RMT, the partition function is given in terms of the joint probability distribution (jpdf) of eigenvalues as

$$
\mathcal{Z}_{n}=\int \prod_{i=1}^{n} d x_{i} w\left(x_{i}\right) \Delta_{n}(x)^{2}
$$

The Vandermonde determinant in the integrand,

$$
\Delta_{n}(x)=\operatorname{det}_{1 \leq i, j \leq n}\left[x_{i}^{j-1}\right]=\operatorname{det}_{1 \leq i, j \leq n}\left[p_{j-1}\left(x_{i}\right)\right],
$$

can be replaced by a determinant over an arbitrary set of monic polynomials. If we choose the orthogonal ones we can rewrite the jpdf and thus the partition function after few manipulations as

$$
\mathcal{Z}_{n}=\int \prod_{i=1}^{n} d x_{i} h_{i-1} \operatorname{det}_{1 \leq j, k \leq n}\left[K_{n}\left(x_{j}, x_{k}\right)\right]
$$

It immediately follows from Dyson's theorem that $\mathcal{Z}_{n}=n ! \prod_{i=1}^{n} h_{i-1}$. Moreover, following eq. (1.9) all $k$-point eigenvalue correlation functions given by $n-k$ integrations over the jpdf can be immediately read off:

$$
R_{k}\left(x_{1}, \ldots, x_{k}\right) \equiv \frac{1}{(n-k) !} \int \prod_{i=k+1}^{n} d x_{i} \operatorname{det}_{1 \leq i, j \leq n}\left[K_{n}\left(x_{i}, x_{j}\right)\right]=\operatorname{det}_{1 \leq i, j \leq k}\left[K_{n}\left(x_{i}, x_{j}\right)\right] .
$$

In the large- $n$ limit the size of the determinant on the rhs remains fixed, and the kernel can be easily evaluated using the Christoffel-Darboux identity for orthogonal polynomials on $\mathbb{R}$.

Since in this example the choice of orthogonal polynomials was entirely at our disposal, why should we choose polynomials that are not orthogonal with respect to the weight function, or the integration range? The reason is that we are not always able to choose the polynomials to be orthogonal. One example where such a situation occurs is in the Schwinger model [2]. A second example, being in a different symmetry class, appears when considering the Ginibre ensemble with real non-symmetric matrices [3]. Integrating out all real eigenvalues one arrives at the Pfaffian of the so-called $D$-kernel of the Gaussian Orthogonal Ensemble [1], integrated over a non-Gaussian weight function in the complex plane. Consequently the self-contracting property eq. (1.1) is not satisfied then.

For this reason we propose a generalisation of Dyson's theorem for determinants without imposing any orthogonality condition, and we restrict ourselves to real integrals for simplicity. The generalisation to integrals over $\mathbb{C}$ is straightforward. A counterpart for integrating Pfaffians of a non self-contracting bilinear has been proved in [3] in the special case when all variables are integrated out.

\section{Results}

Let each $\left\{\phi_{j}(p)\right\}$ and $\left\{\psi_{j}(q)\right\}, j=1, \ldots, n$ be a set of linearly independent2, integrable functions, such that all integrals $\int d x \phi_{i}(x) \psi_{j}(x)$ exist. For these two sets we define the following bilinear function:

$$
Q_{n}(x, y) \equiv \sum_{j=1}^{n} \phi_{j}(x) \psi_{j}(y)
$$

Then the following holds:

\footnotetext{
${ }^{2}$ We note that the functions $\phi_{j}(x)$ may or may not be linear combinations of the functions $\psi_{j}(x)$.
} 
THEOREM 1:

$$
\frac{1}{C} \int \prod_{l=1}^{n-k} d x_{l} \operatorname{det}_{1 \leq i, j \leq n}\left[Q_{n}\left(p_{i}, q_{j}\right)\right]=(n-k) ! \operatorname{det}_{n-k+1 \leq i, j \leq n}\left[\mathcal{K}_{n}\left(p_{i}, q_{j}\right)\right], \quad k=0, \ldots, n,
$$

where we have set $p_{i}=q_{i}=x_{i}, i=1, \ldots, n-k$ for all integration variables.

The kernel $\mathcal{K}_{n}(p, q)$ on the rhs is given by



where the sum runs over the $a$-th column replacing the integrated functions by unintegrated ones. The normalisation $C$ on the left hand side (lhs) is given by

$$
C \equiv \operatorname{det}\left[\begin{array}{llr}
\int d x \phi_{1}(x) \psi_{1}(x) \cdots & \cdots \int d x \phi_{1}(x) \psi_{n}(x) \\
\cdots & & \\
\int d x \phi_{n}(x) \psi_{1}(x) & \cdots & \cdots \int d x \phi_{n}(x) \psi_{n}(x)
\end{array}\right]
$$

Thus we have reduced an $(n-k)$-fold integral over an $n \times n$ determinant to a $k \times k$ determinant of a single kernel, consisting of a sum of $n \times n$ determinants containing only single integrals.

The rhs of our Theorem 1 can be interpreted as a generalised kernel having $2 k$ variables. If we define

$$
\mathcal{K}_{n}^{(k)}\left(p_{1}, \ldots, p_{k} ; q_{1}, \ldots, q_{k}\right) \equiv \frac{1}{k !} \operatorname{det}_{1 \leq i, j \leq k}\left[\mathcal{K}_{n}\left(p_{i}, q_{j}\right)\right]
$$

these satisfy the following generalised self-contraction property (see eq. (1.1)):

\section{THEOREM 2:}

$$
\begin{aligned}
\int d q_{1} \ldots d q_{k} \mathcal{K}_{n}^{(k)}\left(p_{1}, \ldots, p_{k} ; q_{1}, \ldots, q_{k}\right) \mathcal{K}_{n}^{(k)}\left(q_{1}, \ldots, q_{k} ; r_{1}, \ldots, r_{k}\right) & =\mathcal{K}_{n}^{(k)}\left(p_{1}, \ldots, p_{k} ; r_{1}, \ldots, r_{k}\right), \\
\int d q_{1} \ldots d q_{k} \mathcal{K}_{n}^{(k)}\left(q_{1}, \ldots, q_{k} ; q_{1}, \ldots, q_{k}\right) & =\left(\begin{array}{c}
n \\
k
\end{array}\right) .
\end{aligned}
$$

In particular the kernel defined in eq. (2.3), $\mathcal{K}_{n}^{(k=1)}(p ; q) \equiv \mathcal{K}_{n}(p ; q)$, is self-contracting.

Let us make a few remarks. First, the bilinear $Q_{n}(x, y)$ of the set of functions is in general different from the kernel on the rhs: $Q(x, y) \neq \mathcal{K}_{n}(x, y)$. In particular it is not self contractive in general: $\int d y Q(x, y) Q(y, z) \neq Q(x, z)$. In the case of orthogonal functions, $\int d x \phi_{k}(x) \psi_{j}(x)=\delta_{k j}$, we obviously get back $Q_{n}(x, y)=\mathcal{K}_{n}(x, y)$. Then Dyson's theorem applies, as in the example in the previous section.

Special cases of Theorem 1 were previously known. For $k=0$ it goes back to C. Andréief in 1883 as cited in [4], after multiplying with the normalisation $C$ :

$$
\int \prod_{l=1}^{n} d x_{l} \operatorname{det}_{1 \leq i, j \leq n}\left[\phi_{j}\left(x_{i}\right)\right] \operatorname{det}_{1 \leq i, j \leq n}\left[\psi_{j}\left(x_{i}\right)\right]=n ! \operatorname{det}_{1 \leq i, j \leq n}\left[\int d x \phi_{i}(x) \psi_{j}(x)\right] .
$$

The identity for $k=1$ was stated and used in [2] but no explicit proof was given. Furthermore let us point out that for $k=n$ there are no integrations, thus equating the determinant of the bilinear function and of the kernel.

All statements above also hold when the normalisation eq. (2.4) accidentally vanishes, $C=0$, as will be indicated below. This cannot happen for Dyson's integration theorem. 


\section{Proofs}

The proof of Theorem 1 will go in three steps, taking the known result for $k=0$ for granted. In step i) we prove the Theorem for $k=1$, relating to the definition (2.3). In step ii) we show that the kernel $\mathcal{K}_{n}(p, q)$ satisfies the self-contraction property eq. (2.6), Theorem 2 for $k=1$. In the last step iii) we prove Theorem 1 for $k=n$ without integrations. Because of the self-contraction property of $\mathcal{K}_{n}(p, q)$ we can then apply Dyson's theorem to the rhs to show all the remaining cases. Theorem 2 for $k \geq 2$ will then be shown in the second part.

step i): To prove $k=1$, in a first trivial step we can replace the determinant of the bilinear function as follows:

$$
\operatorname{det}_{1 \leq i, j \leq n}\left[Q_{n}\left(p_{i}, q_{j}\right)\right]=\operatorname{det}_{1 \leq i, j \leq n}\left[\sum_{a=1}^{n} \phi_{a}\left(p_{i}\right) \psi_{a}\left(q_{j}\right)\right]=\underset{1 \leq a, i \leq n}{\operatorname{det}}\left[\phi_{a}\left(p_{i}\right)\right] \operatorname{det}_{1 \leq a, j \leq n}\left[\psi_{a}\left(q_{j}\right)\right] .
$$

Inserting this into the lhs we can expand both determinants with respect to the last, unintegrated column:

$$
\begin{aligned}
\frac{1}{C} \int \prod_{l=1}^{n-1} d x_{l} \operatorname{det}_{1 \leq a, i \leq n}\left[\phi_{a}\left(p_{i}\right)\right] \operatorname{det}_{1 \leq a, j \leq n}\left[\psi_{a}\left(q_{j}\right)\right] & =\frac{1}{C} \int \prod_{m=1}^{n-1} d x_{m}\left(\sum_{j=1}^{n} \phi_{j}\left(p_{n}\right) C_{j}^{\phi}\right)\left(\sum_{l=1}^{n} \psi_{l}\left(q_{n}\right) C_{l}^{\psi}\right) \\
& =\frac{(n-1) !}{C} \sum_{l, j=1}^{n} \phi_{j}\left(p_{n}\right) \psi_{l}\left(q_{n}\right) C_{l j} \\
& =(n-1) ! \mathcal{K}_{n}\left(p_{n}, q_{n}\right)
\end{aligned}
$$

where $p_{i}=q_{i}=x_{i}$ for $i=1, \ldots, n-1$. We have introduced the minors

$$
C_{j}^{\phi} \equiv(-)^{n-1+j} \operatorname{det}_{i \neq j}\left[\phi_{i}\left(x_{k}\right)\right] \text { and } C_{l}^{\psi} \equiv(-)^{n-1+l} \operatorname{det}_{i \neq l}\left[\psi_{i}\left(x_{k}\right)\right] \text {. }
$$

These contain $n-1$ functions each, and all variables $x_{1}, \ldots, x_{n-1}$ are integrated. Thus for each product $C_{j}^{\phi} C_{l}^{\psi}$ we can apply the formula for $k=0$ by C. Andréief eq. (2.7), with the resulting minor

$$
C_{l j} \equiv(-)^{l+j} \operatorname{det}_{i \neq j ; k \neq l}\left[\int d x \phi_{i}(x) \psi_{k}(x)\right] \text {. }
$$

In the last step the sum in eq. (3.9) can be precisely written as the sum over determinants in eq. (2.3), each expanded with respect to the $a$-th column.

step ii): To derive the self-contraction property for $\mathcal{K}_{n}(p, q)$ we simply insert the definition eq.(2.3),

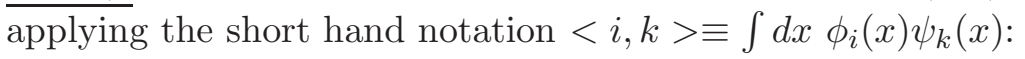

$$
\begin{aligned}
& \int d q \mathcal{K}_{n}(p, q) \mathcal{K}_{n}(q, r)=\frac{1}{C^{2}} \int d q\left(\sum_{a=1}^{n} \operatorname{det}\left[\begin{array}{ccc}
<1,1>\cdots & \phi_{1}(p) \psi_{a}(q) & \cdots<1, n> \\
\cdots & \cdots & \\
<n, 1>\cdots & \phi_{n}(p) \psi_{a}(q) & \cdots<n, n>
\end{array}\right]\right) \\
& \times\left(\sum_{b=1}^{n} \operatorname{det}\left[\begin{array}{lcr}
<1,1>\cdots & \phi_{1}(q) \psi_{b}(r) & \cdots<1, n> \\
\cdots & \cdots & \cdots \\
<n, 1>\cdots & \phi_{n}(q) \psi_{b}(r) & \cdots<n, n>
\end{array}\right]\right) \\
& =\frac{1}{C^{2}}\left(\sum_{a=1}^{n} \operatorname{det}\left[\begin{array}{lcr}
<1,1>\cdots & \phi_{1}(p) \psi_{b=a}(r) & \cdots<1, n> \\
\cdots & \cdots & \cdots \\
<n, 1>\cdots & \phi_{n}(p) \psi_{b=a}(r) & \cdots<n, n>
\end{array}\right]\right) \times C \\
& =\mathcal{K}_{n}(p, r) \text {. }
\end{aligned}
$$


Here we simply observe that in each product of determinants the common factors $\psi_{a}(q)$ and $\psi_{b}(r)$ can be taken out of the columns $a$ and $b$ respectively, and can then be multiplied into the columns of the other determinant. In a second step the integral $\int d q$ can now be taken inside the $b$-th column of the second determinant containing $\phi_{j}(q) \psi_{a}(q)$, resulting into $\langle j, a\rangle$. This leads to a column already present and thus a vanishing determinant, unless we have $a=b$. The resulting normalisation cancels one power of $C$ to reproduce the kernel.

step iii): We prove Theorem 1 for $k=n$ when all integrations are absent. It is easily seen when expanding the kernel inside the determinant on the rhs, using the formula in the last line of eq. (3.9):

$$
\begin{aligned}
\operatorname{det}_{1 \leq i, j \leq n}\left[\mathcal{K}\left(p_{i}, q_{j}\right)\right] & =\operatorname{det}_{1 \leq i, j \leq n}\left[\frac{1}{C} \sum_{l, k=1}^{n} \phi_{k}\left(p_{i}\right) \psi_{l}\left(q_{j}\right) C_{l k}\right] \\
& =\operatorname{det}_{1 \leq i, k \leq n}\left[\phi_{k}\left(p_{i}\right)\right] \operatorname{det}_{1 \leq l, j \leq n}\left[\psi_{l}\left(p_{j}\right)\right] \operatorname{det}_{1 \leq l, k \leq n}\left[\frac{1}{C} C_{l k}\right] \\
& =\frac{1}{C} \operatorname{det}_{1 \leq i, j \leq n}\left[Q_{n}\left(p_{i}, q_{j}\right)\right] .
\end{aligned}
$$

In the first step we used that the determinant of the matrix product is the product of the determinants. Furthermore, the minors are just the matrix elements of the inverse matrix, $C_{l k} / C=\left(C^{-1}\right)_{l k}$, and we used eq. (3.8). Because of ii) we can now apply Dyson's integration theorem to the determinant of the self-contracting kernel $\mathcal{K}_{n}\left(p_{i}, q_{j}\right)$. Using its normalisation, $\int d q \mathcal{K}_{n}(q, q)=n$ which can be trivially seen, we arrive at Theorem 1 for all $k=0, \ldots, n$.

Finally we deal with the case $C=0$. The Andréief formula eq. (2.7) still holds and vanishes identically. To make Theorem 1 nonsingular we multiply it with $C^{k}$. Step i) proving $k=1$ remains true and in general nonzero, using eq. (2.7) for size $n-1$. For $k \geq 2$ the lhs of Theorem 1 times $C^{k}$ is zero. If the matrix under the determinant $C$ eq. (2.4) has rank $n-2$ or less, all determinants inside the kernel eq. (2.3) vanish and make the rhs identically zero. For rank $n-1$ it is easy to see that the kernel eq. (2.3) factorises into two functions of $p$ and $q$, thus having a vanishing determinant on the rhs.

It remains to prove Theorem 2 for $k \geq 2$. The proof goes as follows. On the rhs of (2.6) we substitute each of the two kernels $\mathcal{K}_{n}^{(k)}$ as a determinant of single kernels from their definition (2.5). Using the standard representation of a determinant, we get:

$$
\text { lhs }=\frac{1}{(k !)^{2}} \int d q_{1} \ldots d q_{k} \sum_{\sigma, \sigma^{\prime}}(-1)^{\sigma+\sigma^{\prime}} \prod_{i=1}^{k}\left[\mathcal{K}_{n}\left(p_{\sigma(i)}, q_{i}\right) \mathcal{K}_{n}\left(q_{i}, r_{\sigma^{\prime}(i)}\right)\right] .
$$

The fact that $\mathcal{K}_{n}(x, y)$ is self-contractive allows us to do all the integrals over $q_{i}$, to obtain

$$
\text { lhs }=\frac{1}{(k !)^{2}} \sum_{\sigma, \sigma^{\prime}}(-1)^{\sigma+\sigma^{\prime}} \prod_{i=1}^{k} \mathcal{K}_{n}\left(p_{\sigma(i)}, r_{\sigma^{\prime}(i)}\right)=\frac{1}{k !} \operatorname{det}_{1 \leq i, j \leq k} \mathcal{K}\left(p_{i}, r_{j}\right) \equiv \mathcal{K}_{n}^{(k)}\left(p_{1}, \ldots, p_{k} ; r_{1}, \ldots, r_{k}\right)
$$

The normalisation of the generalised kernel $\mathcal{K}_{n}^{(k)}$ follows directly from the normalisation of the single kernel $\mathcal{K}_{n}(p, q)$ which is $n$, and the repeated application of Dyson's integration theorem to a $k \times k$ determinant integrated $k$ times. This way we get the binomial coefficient:

$$
\int d q_{1} \ldots d q_{k} \mathcal{K}_{n}^{(k)}\left(q_{1}, \ldots, q_{k} ; q_{1}, \ldots, q_{k}\right)=\frac{n !}{(n-k) ! k !} .
$$

The same arguments as at the end of the previous proof apply for $C=0$. 


\section{Conclusions}

We have shown how to reduce any number of integrations over a determinant of a bilinear function of non-orthogonal functions to a smaller determinant of a self-contracting kernel containing only single integrals. This makes the large- $n$ limit feasible in such a general setting at least in principle, given the single integrals can be evaluated. Due to the fermionic nature of the Vandermonde determinant other applications than the mentioned Schwinger model should exist. Our result gives hope that an analogous Pfaffian integration theorem with some variables unintegrated also exists.

After writing up this paper we learned from P. Forrester that the first part of our result was derived independently by Rains [5] in the context of symmetrised increasing subsequences. His alternative proof is formulated in terms of the Pfaffian of an antisymmetric matrix kernel. In contrast, our proof illuminates the close relation to Dyson's theorem.

Acknowledgements: We would like to thank T. Guhr and E. Kanzieper for interesting comments, P. Forrester for pointing out references, and the Referee for his comment on the degenerate case. Part of this work was written up and presented during the workshop on "Random Matrix Theory: Recent Applications" at the Niels Bohr Academy in May 2007, and we thank the organisers for the stimulating atmosphere. Financial support by EPSRC grant EP/D031613/1 (G.A. and L.S.) and European Community Network ENRAGE MRTN-CT-2004-005616 (G.A.) is gratefully acknowledged.

\section{References}

[1] M.L. Mehta, Random Matrices, Academic Press, Third Edition, London 2004.

[2] L. Shifrin and J. J. M. Verbaarschot, Phys. Rev. D 73 (2006) 074008 arXiv:hep-th/0507220.

[3] G. Akemann and E. Kanzieper, arXiv:math-ph/0703019; E. Kanzieper and G. Akemann, Phys. Rev. Lett. 95 (2005) 230201 arXiv:math-ph/0507058.

[4] C. Tracy and H. Widom, J. Stat. Phys. 92 (1998) 809 [arXive:solv-int/9804004]

[5] E. M. Rains, "Correlation functions for symmetrized increasing subsequences", arXiv:math/0006097v1 [math.CO] 